\title{
Establishing a Qualitative Data Archive in Austria
}

\author{
by Andrea Smioski
}

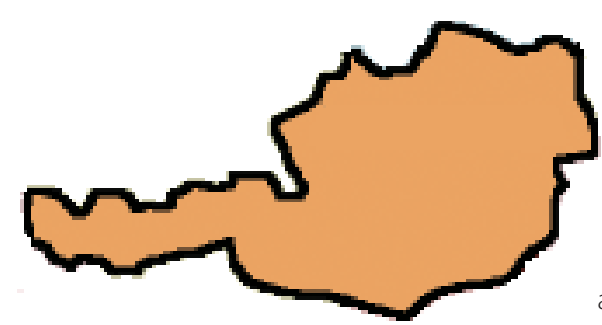

up an archive for qualitative data. $A$

feasibility study was conducted to evaluate the number and condition of available datasets. Moreover, researchers were surveyed about their willingness to deposit and use archived data. This study as well as a following project, aimed at transferring data from the Faculty of Social Sciences at the University of Vienna to the archive, show that researchers have serious concerns about sharing and archiving data. Ethical and methodological questions

were raised. In addition, obstacles such as time-consuming data preparation and the agreement of funding agencies were mentioned.

Since there exists neither an archiving policy in Austria that obliges researchers to deposit their data in a repository nor a culture of data sharing, we are confronted with many obstacles that have to be overcome. Our successes and challenges in starting a qualitative archive shall be described here in greater detail.

Keywords: qualitative data, archiving, data documentation, secondary analysis, establishment of a qualitative archive

\section{Background}

The documentation and archiving of social science research data in Austria started in the 1980s. In 1985, Anton Amann and Anselm Eder, members of the Institute of Sociology at the University of Vienna, Ernst Gehmacher (Institute for Empirical Social Studies, IFES) and Heinz Kienzl (Austrian National Bank), founded WISDOM, the Wiener Institute for Social Science Data Documentation and Methods (WISDOM, n.d). The Ministry of Science and Research supported these efforts. In close cooperation with the University of Vienna and Austrian commercial institutes for market and social research, such as IFES and the Fessel Institute for Market Research, they started to acquire, process and distribute social science survey data. In the 1980s it was relatively easy to acquire data. Bureaucratic and administrative burdens were low and data protection did not pose noteworthy problems. Since then WISDOM has acquired 868 quantitative datasets. More than 480 are adequately processed and documented. They are easy to access in an online catalogue and available for secondary users in digital format. In addition to smaller studies WISDOM holds major national and international public opinion surveys such as the Austrian Social Survey and the Microcensus, which are repeated at regular intervals. Other datasets, such as the ISSP2 and

\section{WISDOM holds major national and international public opinion surveys}

the Eurobarometer ${ }^{3}$ are also available at WISDOM. In the early 1990s WISDOM became a member organisation of CESSDA, the Council of European Social Science Data Archives and thereby became the acknowledged national social science data repository in Austria. Furthermore, in 2001 WISDOM became the national co-ordinator for the European Social Survey (ESS).

In 1984 Bettina Schmeikal established the Social Science Information Center (SOWIS) at the library of the Vienna University of Economics and Business. SOWIS operated a database with detailed information about social science research projects in Austria. In 2006 WISDOM incorporated SOWIS under the new name Research Documentation Social Sciences (FODOS) ${ }^{4}$, which also feeds data into the German-language documentation database of the Leibniz Institute for the Social Sciences (GESIS, n.d). Finally, in 2007 WISDOM began to extend the scope of acquired data to also include qualitative datasets and datasets with mixed 
method designs. This required different expertise and standards. Tools for data management had to be adapted or newly designed. Data protection as well as confidentiality issues had to be addressed in response to researchers worries and fears in relation to qualitative data archiving. These had been revealed in the course of a feasibility study conducted by WISDOM in 2008. Until now and even with joint efforts with the Faculty of Social Sciences of the University of Vienna it has proven difficult to acquire qualitative datasets. Besides confidentiality issues and the fear of misuse, data has come to be regarded as the personal property of researchers or institutions. Due to increasing scientific competition, they are unwilling to share their data with other researchers or institutions. WISDOM's goal is to acquire as many datasets as possible and provide high quality data and meta-data in digital format for secondary users. Furthermore, a dialogue with the research community is promoted through personal contacts, workshops and teaching courses.

This paper presents the data archive WISDOM with a focus on the qualitative part of the archive. Initial steps to establish a qualitative archive and accompanying problems, the results of the feasibility study, and ongoing acquisition efforts and the resulting development strategy will be discussed in detail.

\section{The feasibility study ${ }^{5}$}

In the field of the social sciences, qualitative research has grown more important over the last decades. The long-standing image of qualitative research as the weaker cousin of quantitative research is fading and qualitative research is regarded more and more as an independent research strategy that can reveal aspects of the social world that are inaccessible with only quantitative methods.

Since the 1970s there has existed a broad range of literature on methods of secondary analysis of quantitative data. Important quantitative datasets are routinely stored in data repositories or archives where the data is adequately processed and documented for further reuse. It is common practice to re-analyse and re-consult quantitative research data for different purposes than those in the original research context. Since there are no archives for qualitative data, this tradition of data archiving and re-use is scarcely existent or even possible for qualitative research, at least not in Austria.

The improved image of qualitative research has led to more qualitative research being conducted. A resulting benefit is a growing amount of research material. However, these increasing supplies of qualitative research data are usually stored at offices, researchers' homes or worse, lost in the "data cemetery" and therefore more often than not unexploited and un-used after the initial enquiry. As a consequence, there exists practically no culture of re-use, and new studies seldom build on the empirical results of previous studies to gain deeper insight. Qualitative longitudinal studies or comparison studies are virtually impossible. Researchers have to reinvent the wheel over and over again.

However, in spite of all this there have been some positive developments. ESDS Qualidata, a British qualitative data archive, together with other qualitative archives, tries to nurture a culture of sharing and reusing qualitative research data in Europe. They have already achieved some successes, most notably by supporting the data archiving policy that obliges leaders of projects funded by the Economic and Social Research Council (ESRC) to offer their data-regardless of format-to an archive. Austria, amongst other countries, is trying to take its first tentative steps in a similar direction.
We started with an evaluation of international achievements in the field of qualitative data archiving. We tried to assess which archiving tools and standards could be applied from quantitative to qualitative data, what standards could be easily introduced that take into account the particular needs of qualitative data, and which resources and existing expertise could be adapted. The next step was to raise funds to finance a feasibility study. Finally in 2008, the Austrian Federal Ministry of Science and Research provided the means to conduct this study. The goal was to assess the possibilities of and chances for starting a qualitative social science data archive based on WISDOM's existing infrastructure and experience with data archiving. A quantitative online survey provided an overview of the amount and types of social science research data in Austria, as well as researchers' views about depositing their data in an archive and using archived data for secondary analysis. Expert interviews and workshops deepened the insights gained through the quantitative survey (Müller et al. 2008).

The online survey was targeted at researchers who had done qualitative or mixed methods projects. The design of the questionnaire was largely based on a similar study conducted by the German Central Archive for Empirical Social Science Research at the University of Cologne and the Archive for Life Course Research at the University of Bremen in 2003/04 (Opitz and Mauer 2005). We divided the questionnaire into three connected parts: (1) available data resources (2) secondary use of qualitative data and (3) interest in a qualitative data archive in Austria. The questionnaire was available from March to June 2008 at the WISDOM homepage. We alerted researchers to the questionnaire through mailing-lists; 183 of the 386 responses received could be used for the analysis.

The results showed that interviews and images are the most important data sources for qualitative researchers in Austria. Moreover, they revealed that of all the project data (information on about 1.097 projects conducted since 2000) $56,1 \%$ are stored at offices, $25,9 \%$ at researchers' homes and only 7,9\% in an appropriate archive. 6,5\% of the data are already lost ${ }^{6}$. As a consequence, the accessibility rate is very low, around $10 \%$ for all kinds of data, even lower for interview data, group discussions or data from observations.

Important criteria for archiving and reuse are the condition of data documentation and the data format. One-third of the data is available in digital format, one-third in printed format and the last third in audio and video format. Only about $45 \%$ of the data are sufficiently documented (background and project information) and processed (transcribed and made anonymous). This implies a lot of additional work remains to be done if data are to be archived. The archive can take on some of this work, but as a matter of course we are dependent on the support of researchers to manage and document their data comprehensively, especially in the future when the archive expands from its early stages and more and more datasets will be deposited.

In relation to the secondary use of qualitative research data the analysis revealed that over $60 \%$ of the respondents already re-used their own data and almost $40 \%$ used their colleagues' data. This was quite surprising, since there is very little discussion of secondary analysis of qualitative data in the literature on methods. Another extremely positive result was that over $90 \%$ of the respondents are willing to re-use data in the future and will take the secondary use of archived datasets into consideration for future research projects. The benefits of secondary analyses such as the possibility of comparing datasets, presenting a starting point for further analysis, simplifying interdisciplinary exchange, the possibility of extensive use of otherwise unexploited data, and meta-studies or methods development are all deemed very 
important. As a next step, we asked researchers for the conditions that would have to be fulfilled to make data attractive for re-use. Most important were the guarantee of data quality and the application of standardised quality criteria. Other priorities such as complicance with data protection, extensive documentation and the processing of data, as well as easy access to digital data were also mentioned.

Concerning the interest in a qualitative data archive in Austria the overall picture was encouraging. Researchers show willingness to archive their qualitative data. $77,8 \%$ of the interviewed researchers think their data to be highly suitable for secondary use, $70,7 \%$ of the researchers can imagine depositing research data from finished and ongoing projects in an archive and more than $80 \%$ can imagine archiving data from future projects.

Nevertheless, most researchers would agree to deposit their data in an archive only under certain conditions. They want to know when and how the data is to be used by secondary users and some would like to have the opportunity to co-operate with users of their data. Moreover, researchers want assurance that secondary use would be limited to bona fide researchers conducting academic research. Informed consent of research subjects and anonymisation are perceived as crucial for archiving project data. The agreement of funding agencies, correct citation, monetary compensation and no interference with their own subsequent use of the data are also stated as important factors in the decision to archive data.

However, even if all these conditions are fulfilled, scientists still have reservations that might in the end deter them from actually depositing their data. The quantitative analysis revealed data protection issues and the fear of misuse as the most important objections against archiving qualitative data. Also scientific competition is ranked very high as an impediment. Other reasons concern context sensitivity and specificity of qualitative data. Lastly, also, the funding agencies as data owners and the amount of work involved in preparing the data for archiving are mentioned as hindrances.

Qualitative interviews with experts confirmed and deepened our understanding of these survey results. Again, data protection, data context and documentation, as well as data quality were the highest priorities. Time and money for data processing was another major element of the discussions. The interviewees expressed a need for regulations and restrictions to be imposed on the archive in order to overcome their concerns. Trust was an important topic that showed through all the interviews. There is no accepted and well-known archive for qualitative data, and the necessary culture for data sharing has not yet been established. Therefore, researchers still lack the confidence in such a project.

This result was substantiated by the experiences we gained in three workshops conducted in the university towns Vienna, Graz and Salzburg. Participants strongly supported the idea of an archive for qualitative data but when the question of the location of this archive came up, all of them promoted small local infrastructures that already existed. They felt they would not lose control over their data if they kept them "near" and in archives they had already come to trust. As a result they all opted for decentralized archives under the umbrella of a central institution which would coordinate and manage the data inventory and offer centralised access to the data.

The conclusions that were drawn from the results of the feasibility study for the further course of action comprised: (1) defining key aspects of qualitative archiving, for example depending on the type of data with regard to content, format, qualitative/ quantitative, etc., (2) developing criteria for the quality evaluation of the data, (3) utilising experiences from quantitative data archiving at WISDOM as well as existing international qualitative data archives to develop standards fo qualitative data documentation, (4) addressing and debating issues of confidentiality and data protection on a broader level, (5) establishing cooperation between WISDOM and decentralized local data archives to better address researchers all over the country, (6) intensifying knowledge and offering courses on qualitative data archiving and reuse, and (7) advocating changes in national policies to support data archiving and sharing.

\section{The current situation for acquiring and sharing data in Austria}

The data acquisition policies of existing archives support the principles of data sharing and open access to research data. Data sharing is expected to advance research and scientific ethics, quality of research and learning, and to make more efficient use of public funding. However, the level of support for data sharing is uneven among countries (Laaksonen et al. 2006). In Austria there is no national policy on data archiving and sharing and therefore, a research culture for archiving and sharing research data for secondary use has not gained ground so far. There are no legal requirements requiring that research data be transfered to archives, not even in cases where research projects are publicly funded. WISDOM continuously presses this concern in negotiations with ministries and funding agencies but so far to no avail.

Due to the structure of the scientific employment situation many researchers feel utterly overburdened by the combination of research, teaching and administrative tasks. Under these conditions it seems unlikely that an archiving project will become widely accepted without adequate policies on data archiving and sharing. Interviews with researchers revealed that there is hardly any spare time available for preparing and processing the data so they can be archived properly. Even in cases where the idea of archiving is strongly endorsed, researchers feel they do not have the necessary resources in time, money or staff to fulfil the requirements of basic documentation and data processing. Therefore, an archiving project depends mostly on single researchers who are willing to support the project at their own expense.

This results in a very slowly growing pool of archived datasets and thus limited possibilities for researchers who browse the data catalogue for possible datasets to reuse. There are additional implications: researchers find that there are very few datasets available at the archive and after a few unsuccessful tries, they stop relying on the archive as a source of suitable data. Consequently, trust cannot develop and new datasets remain hard to acquire. To put an end to this vicious circle we initiated a second project shortly after completing the feasibility study.

From September 2008 to February 2009 we ran a project in co-operation with the Faculty of Social Sciences of the University of Vienna ${ }^{7}$, which aimed to take stock of and evaluate the growing pool of research data available at the Faculty and to transfer these data to the archive (Müller et al. 2009; Richter et al. 2009)8. Objects of investigation were dissertations and third-party funded projects at the Faculty of Social Sciences. They were examined and evaluated according to their suitability for archiving.

Furthermore, it was hoped that expert interviews with leading professionals from the Faculty would reveal especially suitable datasets and encourage a dialogue between the research community and the archive as well as a discussion about data archiving and sharing in general. Last but not least, measures were to be developed to prevent 
any further loss of data generated at the Faculty and to ensure the future availability of existing qualitative data for research and for teaching purposes.

We started the project by examining existing qualitative and quantitative data resources. Dissertation projects from 2005 to 2009 were listed as well as third-party funded projects of the same time period. Dissertations were divided into three groups according to the methodological approach used:

It is evident that the vast majority of dissertations made use of qualita- archiving policy. Firstly, it could prevent further loss of qualitative research data. Secondly it would enhance transparency and provide good documentation and therefore improve the quality of research and research outputs.

We then contacted the authors of positively evaluated dissertations to negotiate data deposition. At the same time expert interviews and negotiations with project leaders from third-party funded projects took place. Interestingly, the different methodological approaches of the four disciplines at the Faculty seem to result in different attitudes towards data sharing. Social and Cultural Anthropology proved to be a special case since anthropologists mainly use anthropological fieldwork as a research method. Observational methods are more typical and often entail a long term fieldwork and close relationship with research participants. That relationship with the research subject is something very central to anthropological field studies. The transfer of data to someone else, even another researcher, can be considered a breach in confidentiality. Sociologists on the other hand seem to be more familiar with the idea of data archiving and re-using. Some of them have even re-used data themselves. The firmly established tradition of archiving quantitative data in sociology probably causes a greater openness to the idea of sharing qualitative data in contrast to Social and Cultural Anthropology where the focus primarily lies on qualitative methods. It is not surprising that amongst sociologists we found the highest approval of and support for our project. Political scientists often use single expert interviews as foundation or substantiation for their theses. They stand alone and the projects and dissertations were therefore often classified as not suitable for archiving.

Finally, the empirical data we acquired was processed and made available in digital format for secondary users. Since we received not only digital data but also data in printed and audio format, as well as incomplete, un-transcribed and un-anonymised data, this often meant tremendous effort of data processing mainly by the archive. For data description we used the DDI related meta-data standards ${ }^{9}$ we already had in use for the documentation of quantitative data. Obviously, we had to adapt the original information fields to the specifics of qualitative data (e.g., kind of interview data, detailed description of data collection situation, processing information, etc. $\left.^{10}\right)$ and elaborated the confidentiality declaration. Moreover, we developed data deposition forms, end user agreements and comprehensive user guidelines addressing all relevant topics of archiving and sharing research data, especially data protection and confidentiality issues $^{11}$

The success of the project in acquiring data was relatively modest. Altogether, we were able to get data from eight small scale projects, seven qualitative and one quantitative. Even working in co-operation with the Faculty in a data archiving project it was not possible to overcome the reservations many researchers felt towards archiving 
their data. Though most mentioned concerns about data protection as well as confidentiality, first and foremost, too little time for data processing and restrictions by funding agencies impeded actual data deposition. Still a good share of the interviewees emphasized that they would like to browse sample studies and to get more information on data archiving and sharing before depositing "their" data in the archive. This is a clear sign that trust has yet to be established and that researchers have to be encouraged to actively join the growing but still emergent dialogue about data archiving and sharing. On the other hand, we received several unexpected and spontaneous offers of support. We were presented with opportunities to present our work in methods courses and to run workshops at Faculty facilities. This will hopefully help to stimulate this dialogue and motivate researchers to take an active part in the archiving project.

\section{Development planning and strategies for the future}

WISDOM is the only social science infrastructure facility in Austria providing major national and international quantitative studies. Some of them are repeated at regular intervals, for instance the Austrian Social Survey and the Microcensus. Other datasets, such as the ISSP and the Eurobarometer are also available from WISDOM. Concerning qualitative data, WISDOM still has to build up competencies and data stock and gain the trust of the research community in Austria. Despite all the efforts in the co-operative project with the Faculty of Social Sciences at the University of Vienna, it was not possible to acquire and process many datasets. Since the start of the archiving project in September 2008 we collected seven qualitative datasets, three of which are fully processed and accessible in the online catalogue for interested users. They are very small projects with fewer than 20 cases each, and they all used interviewing methods in the data collection process. There are also participant observation notes and letters. The material is available mostly in digital format (Word, PDF); additionally there are audio files (cassette, mini cassette) from some of the interviews. However, it became apparent that although the archived projects were conducted not long ago, all of the studies were missing some materials which were available only in printed format. In light of this, we must assume that a great deal of additional work for processing will be necessary in the future. Until now no qualitative longitudinal studies have been deposited in the archive. As far as we know, only one notable qualitative longitudinal study (by Larcher and Vogel) was conducted in Austria at all ${ }^{12}$

Since we have started archiving qualitative data only very recently, we cannot provide estimates for the number of users yet. Drawing on experiences with quantitative data, we can say that approximately two-thirds of users are students using the data for diploma or dissertation theses. One-third of the data is used by researchers, mainly from a non-university research background, and a good share of them from abroad.

Along with WISDOM there are some centres and institutes across the country, which - besides other activities - also archive and provide qualitative data on a very small scale. These facilities mainly developed around individual researchers or in the context of institutes and departments, many of which have a contemporary history or archival science background. Datasets are often not systematically processed and are made available only after personal query.

The feasibility study revealed that researchers in Austria would prefer depositing their data in existing local repositories. These could be connected by WISDOM as the hub or the focal point, and WISDOM would undertake the responsibility for evaluating, acquiring, processing and documenting data as well as setting access conditions, transferring and publicizing data (Corti 2000). ESDS Qualidata, an established qualitative archive in Britain, used a form of distributed network model (ibid.) when it was initially established. The data were held in different repositories all over the country and distributed to users from the different sites.

We are currently working on a networking project similar to ESDS Qualidata, funded by the Austrian Federal Ministry of Science and Research. The aim is to build up a network of national data archives with WISDOM as the connecting umbrella organization and also to develop common standards and tools for acquiring and documenting data. Moreover, we are creating a shared platform for users with a comprehensive data catalogue and easily accessible, downloadable datasets. The data documentation will also be available in English so that the path is clear for future expansion of the network on an international scale. In the course of networking negotiations we will concentrate our efforts on acquiring new datasets of big and important national qualitative studies that promise to attract a large group of interested re-users.

Besides this ongoing project we constantly struggle to locate additional funding. At the moment WISDOM is financing the qualitative archive solely with project resources from the Austrian Federal Ministry of Science and Research. This allows for one half-time position covering all relevant fields of work: data acquisition, data processing, user support, networking negotiations, training researchers in archival awareness, promoting secondary use of sources and addressing other important issues of qualitative data archiving and sharing in Austria. Considering that no culture for archiving and secondary use is in place and that funding agencies do not see the benefits of policies on data archiving and sharing yet, it results in a full-time workload for a halftime position. WISDOM is in possession of the necessary infrastructure for qualitative archiving. The problem clearly resides in a research tradition that favours the development of isolated research islands (Kozeluh 2008). This tradition explains the missing legal regulations of funding agencies and the missing commitment of social scientists in Austria. At the moment the situation in Austria is one of segregated research islands with everyone inventing the wheel anew, a situation that is probably not going to change, unless the necessary legal foundations are altered.

Provided that the necessary financial resources are available, the following aims are of major importance in the course of the next few years: (1) negotiating contractual terms and conditions for archiving with funding agencies, (2) identifying and evaluating archivable research materials and arranging for their deposit, (3) promoting and encouraging the secondary use of this data, (4) training future researchers in archival awareness, (5) developing best practice on confidentiality, (6) giving advice on copyright of archived research material, and (7) establishing networks with other national and international repositories for social science research data. As we have long tried to convince funding partners of the necessity of legal regulations concerning data archiving, we are aware that this essential step will continue to be challenging. Long established administrative and bureaucratic ways and common procedures are obstacles that are difficult to overcome and the willingness to improve the current situation is startlingly low. This is true not only for funding agencies, but also for researchers. Therefore, the advancement of archival awareness remains an important objective, both of funding agencies and individual researchers. The acquisition of data and thus the possibility for secondary use depend on achieving this goal. Working on issues of copyright and confidentiality is a significant accompanying measure, since these questions are repeatedly raised by prospective data depositors and are not explicitly and extensively covered in the Data Protection Act or Copyright Act (DGS 2000, UrhG BGBI I 81/2006) of the Austrian court 
of law. Finally, this is also the crucial factor determining if further funds can be acquired since it legitimises the importance of our work.

Finally, international cooperation with other social science data archives and repositories might prove important for the adoption of existing expertise and best practice. International cooperation might strengthen claims towards funding agencies and researchers as well as offer the possibility to extend the scope of available data for users. Organisations like IASSIST, CESSDA and the future CESSDA/ERI first and foremost enable networking and the exchange of experience and expertise, which are crucial for the development of this relatively new project of qualitative data archiving. New ideas can be developed jointly, thus permitting the exchange of tools and data. They further assist in strengthening the efforts of national archives by giving weight and legitimation to our efforts to seek funding and increase support from researchers. Without the platforms provided by these organizations, much of the work we do would be destined to remain on a national scale.

\section{References}

Bundesgesetz über das Urheberrecht an Werken der Literatur und der Kunst und über verwandte Schutzrechte (UrhG), StF: BGBI. Nr. 111/1936 i.d.F. BGBI I Nr. 32/2003, 22/2006 und 81/2006

Corti, L. (2000).'Progress and Problems of Preserving and Providing Access to Qualitative Data for Social Research-The International Picture of an Emerging Culture.' Forum: Qualitative Social Research. 1(3). .[Online]. Available at: http://nbn-resolving.de/urn:nbn:de:0114fqs000324 [Accessed August 6, 2009] .

Datenschutzgesetz 2000. DSG 2000. BGBI. I Nr. 165/1999. http://www. dsk.gv.at/site/6229/default.aspx (accessed August 6, 2009).

GESIS. (n.d). 'Leibniz-Institut für Sozialwissenschaften (formerly Gesellschaft Sozialwissenschaftlicher Infrastruktureinrichtungen e.V'. [Online]. Available at: http://www.social-science-gesis.de/ [Accessed August 20th, 2009]

Kozeluh, U. (2008). Struktur der Geistes-, Sozial- und Kulturwissenschaften in Österreich. Bericht im Auftrag des RFTE Rat für Forschung und Technologieentwicklung.

Laaksonen, H, Sami,B and Stebe, J.(2006). Setting up Acquisition Policies for a New Data Archive. IASSIST Quarterly. 30 (1) [Online]. Available at: http://www.iassistdata.org/publications/iq/iq30/iqvol301laaksonen.pdf [Accessed August 3rd, 2009]

Müller, K., Smioski, A and Bischof, C. (2009). 'Archiving of qualitative and quantitative research data at the Faculty of Social Sciences of the University of Vienna'. Research Report 14. [Online]. Available at: http://www.wisdom.at/ [Accessed August 24th, 2009]

Müller, K., Smioski, A, Casado Asensio, J and Kritzinger, S. (2008). 'Feasibility Study for the Preservation and Dissemination of qualitative research data in Austria'. Research Report 12. [Online]. Available at: http://www.wisdom.at/ [Accessed August 24th, 2009]

Opitz, D and Mauer, R. (2005).'Erfahrungen mit der Sekundärnutzung von qualitativem Datenmaterial - Erste Ergebnisse einer schriftlichen Befragung im Rahmen der Machbarkeitsstudie zur Archivierung und Sekundärnutzung qualitativer Interviewdaten'. Forum: Qualitative Social Research. 6(1).[Online]. Available at: http://nbn-resolving.de/ urn:nbn:de:0114-fas0501431 [Accessed August 3rd, 2009).
Richter, R, Smioski, A and Bendl, T. (2009). Archiving of qualitative and quantitative research data at the Faculty of Social Sciences of the University of Vienna. Faculty Research Report: University of ViennaWISDOM. [Online]. Available at: http://www.wisdom.at/ [Accessed August 24th, 2009]

\section{Notes}

1. Andrea Smioski, andrea.smioski@wisdom.at. Qualitative Data and Support Services Manager, Wiener Institute for Social Science Data Documentation and Methods (WISDOM), Vienna, Austria. http:// www.wisdom.at

2. The ISSP is a continuing annual programme of cross-national collaboration on surveys covering topics important for social science research. http://www.issp.org/ (August 20, 2009)

3. Since 1973, the European Commission has been monitoring the evolution of public opinion in the Member States. http://ec.europa. eu/public_opinion/index_en.htm (August 20, 2009)

4. FODOS: FOrschungsDOkumentation Sozialwissenschaften

5. The final report of the feasibility study is available in German and can be ordered.

6. 3,6\% of the data is stored "elsewhere".

7. The Faculty of Social Sciences of the University of Vienna consists of four major study programs: Political Sciences, Communication Sciences, Sociology and Social and Cultural Anthropology. Over 17.000 students are inscribed, which is $20 \%$ of overall amount of students at the University of Vienna. Per year about 50 PhD theses and about 600 MAs are finished.

8. The project was also funded by the Austrian Federal Ministry of Science and Research

9. The Data Documentation Initiative is an international effort to establish a standard for technical documentation describing social science data. See, http://www.icpsr.umich.edu/DDI/ (August 20, 2009)

10.. We geared to the data description information applied to datasets by ESDS Qualidata

11. The user guides were distributed in paperback at the Faculty of Social Sciences. Furthermore these information is available on the new WISDOM homepage that was re-launched by end of August 2009

12. Manuela Larcher and Stefan Vogel used data from 100 in-depth interviews with organic farmers, conducted in the 1990ies by the Department of Sustainable Economic Development at the University of Natural Resources and Applied Life Sciences in Vienna and compared them to recently collected data on the same topic to evaluate changes in household strategies of organic-farms.

13. E.g. the "Documentation of Life History Records Association" at the department of Economic and Social History at the University Vienna, the "Documentation Centre of Austrian Resistance" (DÖW), the "Department for Contemporary History" at the University of Vienna and others.

14. IASSIST: International Association for Social Science Information Service \& Technology. http://www.iassistdata.org/ (August 20, 2009)

15. CESSDA: Council of European Social Science Infrastructures. http://www.cessda.org/ (August 20, 2009), CESSDA/ ERI (European Research Infrastructure) 